\title{
Integrierte Personal- und Organisationsarbeit im Produktionsmanagement
}

A. Personal- und Organisationsarbeit im Rahmen des Produktionsmanagements

1. Standort und Stellenwert des Human-Ressourcen-Managements

2. Verbundformen im Human-Ressourcen-Management

B. Bedarfsverbund im Human-Ressourcen-Management

1. Personalbedarfe im Produktionsmanagement

2. Organisationsbedarfe im Produktionsmanagement

C. Ergänzungsverbund im Human-Ressourcen-Management

1. Entgeltpolitik

2. Kooperation

3. Unternehmertum

4. Ideenmanagement

5. Rationalisierung

6. Technologiefolgen-Management

D. Verdrängungsverbund im Human-Ressourcen-Management

Literatur 


\section{A. Personal- und Organisationsarbeit im Rahmen des Produktionsmanagements}

\section{Standort und Stellenwert des Human-Ressourcen-Managements}

Unterteilt man das Produktionsmanagement in die drei Managementsparten ${ }_{n}$ Pro-

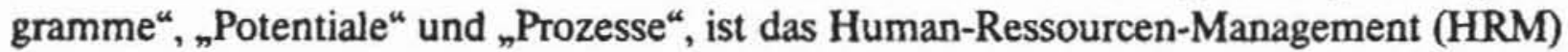
zusammen mit dem Management der technischen Ressourcen (Anlagenwirtschaft und Materialwirtschaft) in der Sparte "Potentialmanagement" anzusiedeln. Das Management der Human-Ressourcen (auch: „Humankapital ${ }^{4}$, „Humanpotential“, , human resources“ bzw. , human assets") vereinigt zwei getrennte Betrachtungsweisen im Produktionsmanagement: zum einen die Ansätze zur Optimierung des Produktionsfaktors Arbeit, also der menschlichen Arbeitsleistung im Produktionsbereich. Zum anderen die Ansätze zur Personalführung, die sich unter anderem mit Führungsstilen, Gruppenarbeit (Zirkel, Lernstatt, Projektgruppen, teilautonome Teams usw.) und Cost-Center-Konzepten im Produktionsbereich befassen. Aktuelle Modelle einer humanzentrierten Produktion, etwa in Form eines „Human Integrated Manufacturing ", gehen mit einer Aufwertung der HumanRessourcen gegenüber den technischen Ressourcen (ooptimaler Automatisierungsgrad “, ${ }_{n}$ dezentral beherrschte Technik" etc.) einher. Das HRM ist so ganzheitlich angelegt, daB einschlägige Managementansätze wie etwa das Personalmanagement, die Arbeits- bzw. Produktionsorganisation, die Arbeitswissenschaft oder das Management der industriellen Arbeitsbeziehungen als Teilgebiete im HRM enthalten sind.

Das Instrumentarium des HRM ergibt sich im Kern aus einer Zusammenlegung aller Instrumente der Personalarbeit (Personalmanagement, Personalwirtschaft) einerseits und der Organisationsarbeit (Organisationsgestaltung) andererseits. Eine Integration dieser beiden Management- bzw. Führungsfunktionen empfiehlt sich vor dem Hintergrund der engen Abhängigkeiten zwischen den beiden Domänen, in denen sich sowohl die Personalals auch die Organisationsarbeit betätigen (vgl. Abbildung 1). Es handelt sich um die Gestaltung der beiden Komplexe "Personal ${ }^{\prime \prime}$ und ${ }_{\text {Arbeit }}$. Diese repräsentieren zugleich gemä $B$ der allgemeinen Verhaltensformel „Verhalten $=$ (Person, Umwelt) ${ }^{4}$ die generischen Determinanten des Arbeitsverhaltens.

Jede Optimierung von Produktionssystemen beruht auch auf einer wechselseitigen $\mathrm{Ab}$ stimmung dieser beiden Einflußgrößen. Erfolg oder Mißerfolg von Restrukturierungsvor-

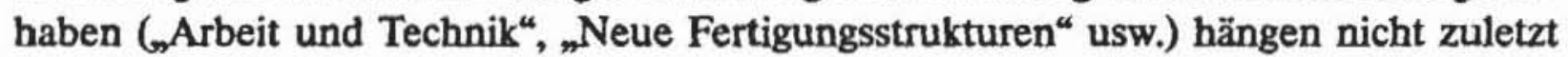
vom Integrationsgrad der verschiedenen organisatorischen und personalpolitischen Einzelprojekte ab.

Die beiden Führungsfunktionen unterscheiden sich hauptsächlich hinsichtlich der Intensität, mit der sie das im Produktionsproze B tätige Personal einerseits bzw. die produktionsseitigen Arbeitsverhältnisse andererseits beeinflußen. Das „Stammgeschä $\mathrm{ft}^{\alpha}{ }^{\alpha}$ des Personalmanagements wird durch die klassischen Personalfunktionen der "Personalgestaltung" definiert (vgl. Abbildung 2). Hierzu zählt beispielsweise die Einflußnahme auf den Führungsstil von Meistern (Personalführung), auf das Leistungspotential des einzelnen Mitar- 


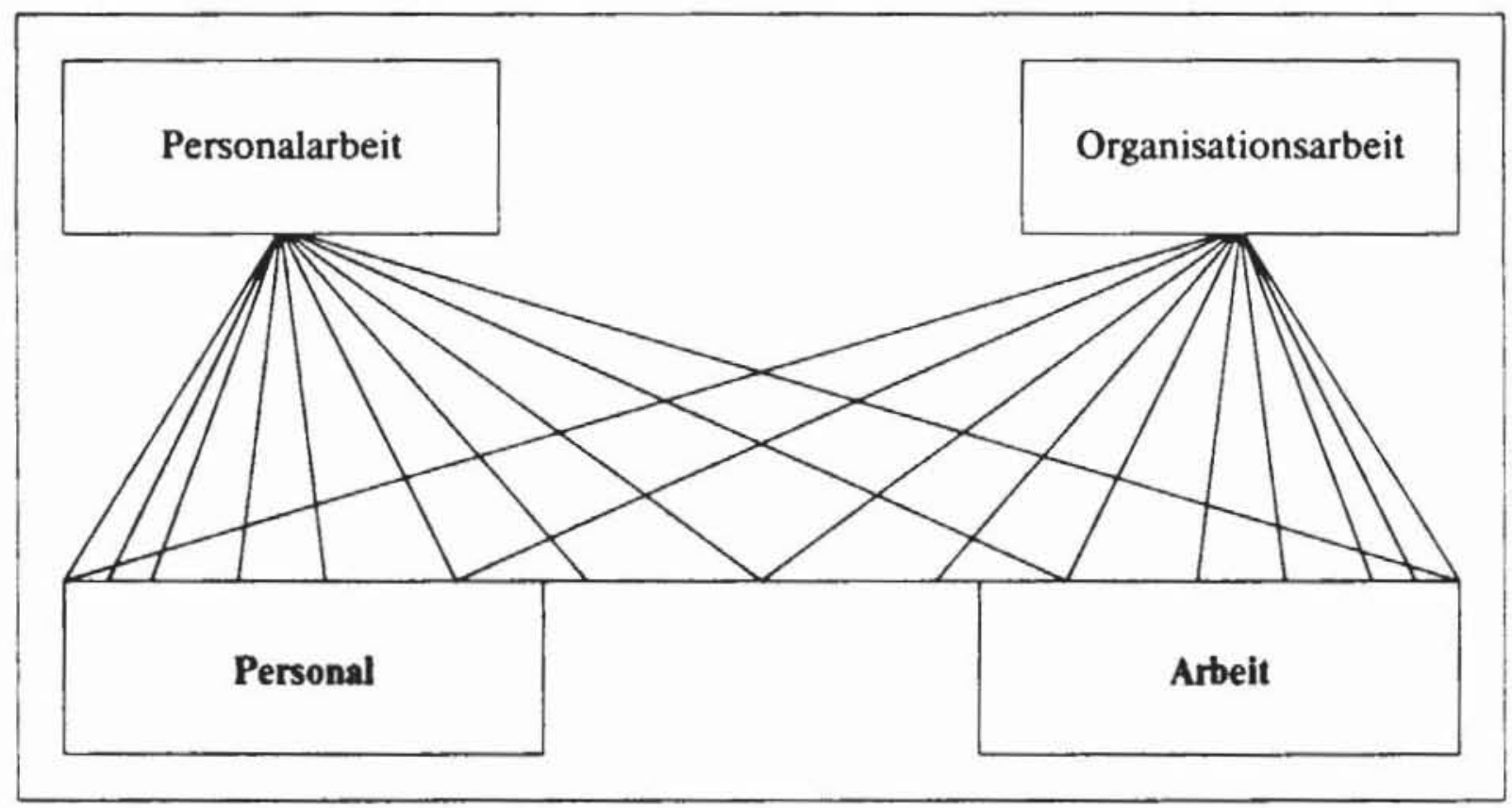

Abbildung 1: Interdependenz von Personal- und Organisationsarbeit

beiters (Erhaltung von Gesundheit und Arbeitseinstellungen, Entwicklung von Qualifikationen), die Rekrutierung von Facharbeitem (Personalbeschaffung auf internen und externen Arbeitsmärkten, Bewerberauswahl), die Vertragsgestaltung (optimale Bindung durch unbefristete bzw. befristete Arbeitsverträge, Werkverträge mit Subunternehmern usw.), die Personalfreisetzung sowie die produktionsinterne Zuordnung (Personaleinsatz nach Einsatzstellen, Einsatzzeiten und Einsatzorten). Darüber hinaus wirkt das Personalmanagement auf die Arbeitsgestaltung ein. Das Spektrum reicht hier von der Einflußnahme auf Arbeitsziele (z.B. Pensumvereinbarungen für Arbeitsgruppen), Arbeitstätigkeiten und -abläufe (z.B. Programmierungs- und Instandsetzungsarbeiten, abwechslungsreiche Tätigkeiten), Arbeitsmittel und-objekte (z.B. Bildschirmarbeit, Teilefamilien), Arbeitszeit- und Arbeitsraum (etwa Schichtplanung, u-förmige Anordnung von Arbeitsplätzen), Gruppenbzw. Einzelplatzarbeit, Gestaltung von Beleuchtungs- und Klimafaktoren bis hin zur Gestaltung von Anreizsystemen (Arbeitsbewertung, Prämienlohnsysteme, Erfolgsbeteiligung, Vorschlagswesen usw.).

Die "Kernkompetenz ${ }^{\prime}$ der Organisationsarbeit liegt auf dem Gebiet der Arbeitsgestaltung (vgl. Abbildung 3). Organisatorische Maßnahmen regeln im Rahmen der Aufbauorganisation die Bildung von organisatorischen Produktionseinheiten (z.B. Werke, Werkstätten, Cost-Center, Fertigungssegmente bzw. -inseln, Service-Zentren). Hier läßt sich eine Tendenz zur Ablösung der funktionsorientierten Werkstattfertigung durch eine objektorientierte Segmentfertigung feststellen. Organisatorische Regelungen betreffen desweiteren die Koordination der einzelnen Produktionseinheiten, hauptsächlich das abgestimmte Zusammenwirken von Querschnittsfunktionen und Leistungsfunktionen sowie von Komponentenfertigung, Vormontage und Endmontage. Analog werden in der Ablauforganisation bestimmte Ablaufeinheiten in Zeit und Raum gebildet. Dazu zählen beispielsweise Losgrößen, Stückprozesse, aber auch standardisierte Einheiten wie beispielsweise Kalenderwochen oder normierte Transporteinheiten. Einige organisatorische Regelungen wie 


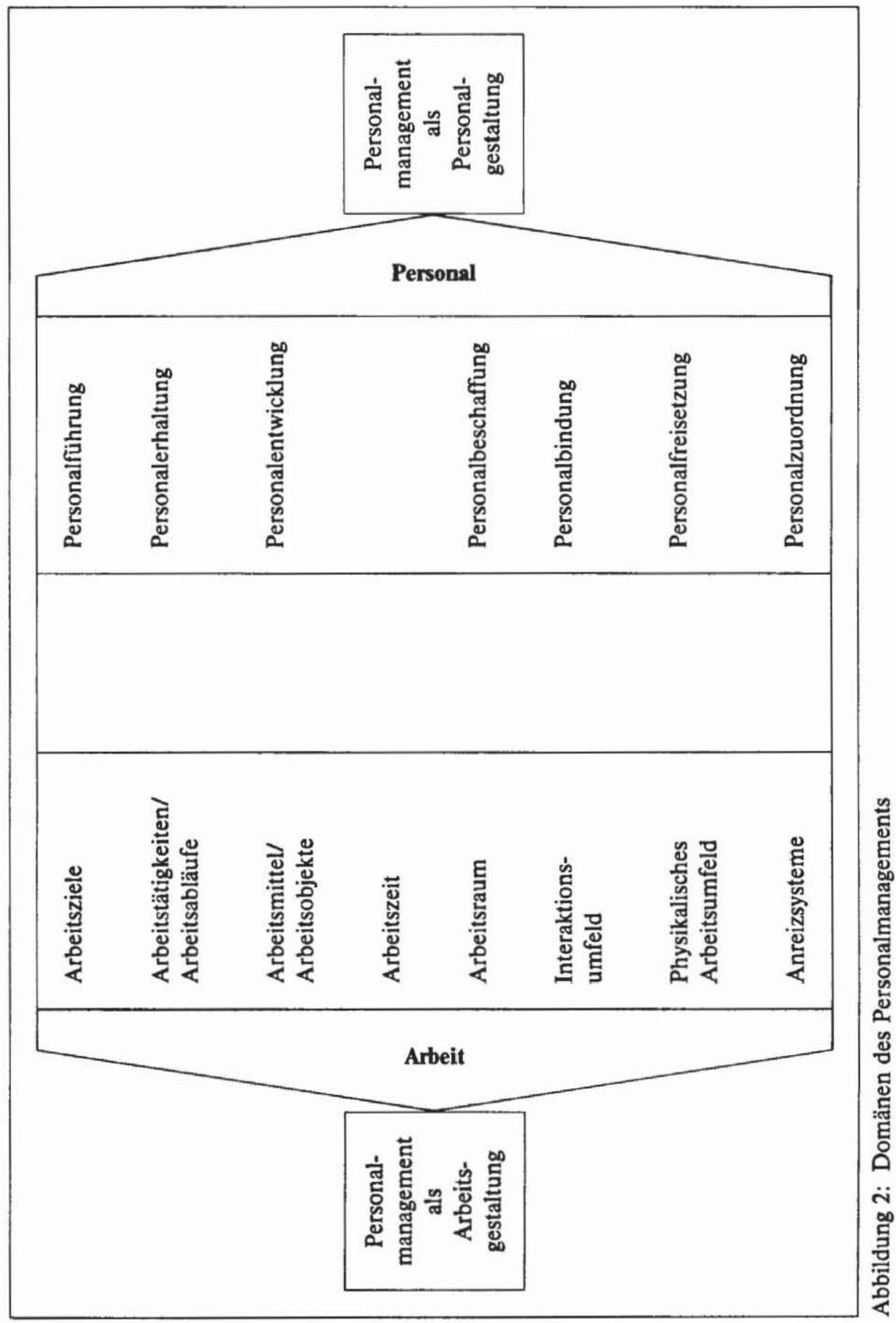




\begin{tabular}{|c|c|c|}
\hline & $\begin{array}{c}\text { Verteilungsstrukturen } \\
\text { (Bildung organisatorischer } \\
\text { Einheiten) }\end{array}$ & $\begin{array}{l}\text { Koordinationsstrukturen } \\
\text { (Abstimmung organisa- } \\
\text { torischer Einheiten) }\end{array}$ \\
\hline $\begin{array}{l}\text { Aufbaustrukturen } \\
\text { (Sachbezogene } \\
\text { Strukturierung) }\end{array}$ & $\begin{array}{l}\text { Aufbaueinheiten } \\
\text { Bereichseinheiten, } \\
\text { Abteilungen, Stellen, } \\
\text { Projektgruppen }\end{array}$ & $\begin{array}{l}\quad \text { Aufbaukoordination } \\
\text { Kommunikation, } \\
\text { Gremienbildung, Anweisung, } \\
\text { Verhandlung, Hierarchie }\end{array}$ \\
\hline $\begin{array}{l}\text { Ablaufstrukturen } \\
\text { (Zeit- und } \\
\text { raumbezogene } \\
\text { Strukturierung) }\end{array}$ & $\begin{array}{l}\text { Ablaufeinheiten } \\
\text { - Lose, Stapel, Chargen, } \\
\text { Prozesse (Vorgangsfolgen) } \\
\text { - Zeiteinheiten } \\
\text { (Perioden, Episoden) } \\
\text { - Raumeinheiten } \\
\text { (Lager- bzw. Speichereinheiten, } \\
\text { Büro- bzw. Fabrikflächen) }\end{array}$ & $\begin{array}{l}\text { Ablaufkoordination } \\
\text { - Parallelisierung, Staffelung, } \\
\text { Synchronisierung, } \\
\text { Emanzipation, } \\
\text { Ortsgleichheit } \\
\text { - Prioritäten, Repetitivität, } \\
\text { Varietät, Rundreisen }\end{array}$ \\
\hline
\end{tabular}

Abbildung 3: Parameter der Produktionsorganisation

etwa die Losgrößenbildung büßen bei flexibel automatisierter Produktion an Bedeutung ein, wenn ohne wesentliche Produktivitätsverluste auch chaotisch produziert werden kann.

Von der Produktionsorganisation gehen auch markante Einflüsse auf die Personalgestaltung aus. In diesem Zusammenhang sei auf das intrinsische Motivationspotential von angereicherten und erweiterten Stellen (zur Aktivierung bzw. Erhaltung von positiven Arbeitseinstellungen), auf die Transparenz von Prozessen durch klare Regelungen (z.B. Prämiengewährung im Vorschlagswesen) und auf das Training von Sozialkompetenzen durch Gruppenarbeit (learning by doing) verwiesen.

Grundsätzlich verfolgt ein integrativ angelegtes HRM auch ein Harmonisierungsanliegen im Spannungsfeld „Unternehmungsziele - Mitarbeiterziele“. Dies wird dadurch ermöglicht, daß das Management der Human-Ressourcen durch Interaktionsmodelle (AnreizBeitrags-Theorie, Equity-Theorie, Arbeitsmarkt- und Transaktionskostenmodelle usw.) abgebildet wird (vgl. Abbildung 4).

Diese Führungsperspektive (besser: Interaktionsperspektive) schlägt sich in Modellen der Personalsozialisation (Integration neuer Mitarbeiter in die Unternehmenskultur), Personalsteuerung (von Leistungs-, Lern- und Interaktionsprozessen) sowie des Personalmarketing nieder. Sie alle tragen der Tatsache Rechnung, daß sich das HRM in einem Spannungsfeld von mehr oder weniger konträren Interessen bewegt. Dieser praktisch äußerst wichtige Aspekt läßt sich im Rahmen der klassischen Bereitstellungsperspektive mit ihren allokationstheoretisch angelegten Modellen des Personaltrainings, des Personaleinsatzes und der Personalrekrutierung nicht adäquat berücksichtigen. 


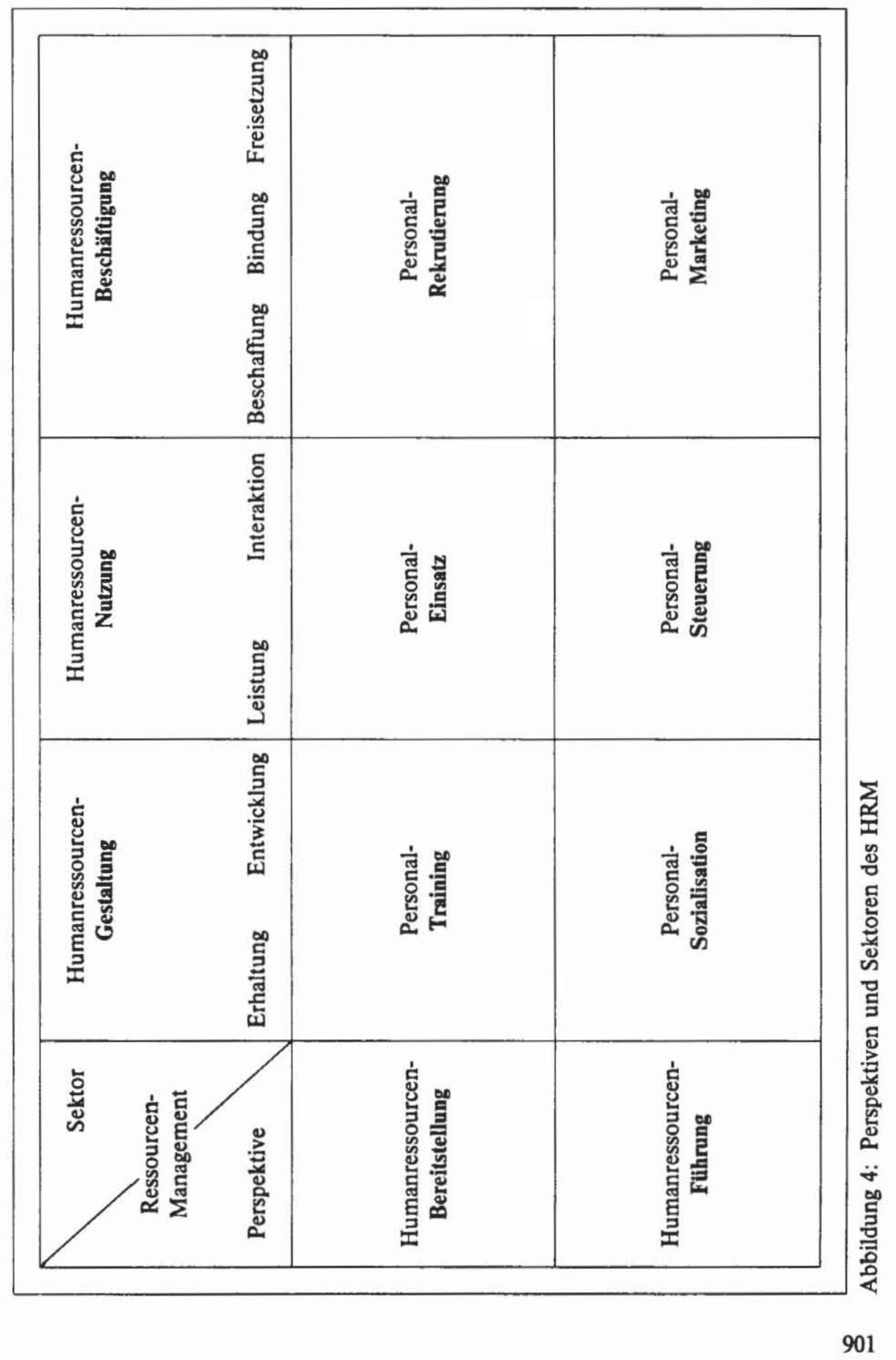


Voreilig wäre es jedoch, aus dieser ${ }_{n}$ formalen “ Berücksichtigung von Mitarbeiterzielen auf eine normative Humanzentrierung des HRM zu schließen. Inwieweit effektiv eine Humanisierung der Produktion verwirklicht wird, hängt unter anderem von der faktischen Machtverteilung auf den Arbeitsmärkten bzw. in den politischen Gremien der Arbeitsgesetzgebung ab.

\section{Verbundformen im Human-Ressourcen-Management}

Das HRM kann nur dann zu Effektivitäts- und Effizienzsteigerungen in der Produktion beitragen, wenn das umfangreiche Instrumentarium der Personal-und Organisationsarbeit in optimalen Konfigurationen zum Einsatz kommt. Ähnlich wie Marketing-Manager bei der Konfigurierung eines optimalen Marketing-Mix müssen sich HR-Manager an den bestehenden Verbundbeziehungen zwischen den einzelnen Parametern orientieren. Konkret sind dabei drei Kategorien von Abhängigkeiten zwischen Personal- und Organisationsinstrumenten zu berücksichtigen: Im Rahmen eines Anpassungsverbunds lösen bestimmte Aktivitäten in einem Bereich direkt Anpassungsnotwendigkeiten in anderen Bereichen aus. Im Brennpunkt steht hier der Bedarfsverbund. Gängige Faustregeln wie z. B. „Personal folgt Organisation" bzw. „Organisation vor Persona!!" dokumentieren die Stringenz eines solchen Bedarfsverbunds. Die aktuelle Relevanz dieses Verbunds ergibt sich aus der immer häufiger erhobenen Fordenung nach Umkehrung der Anpassungsrichtung ( ${ }_{n}$ Personal vor Organisation“) bzw. aus Plädoyers für eine wechselseitige Abstimmung.

Leicht eingängig ist der Grundgedanke des Ergänzungsverbunds zwischen Personal- und Organisationsmaßnahmen. Nur über einen komplementären Einsatz von organisatorischen Regelungen für Prüfprozesse und von Qualifizierungen auf den Gebieten Statistische ProzeB-Kontrolle (SPC), Fehler-Möglichkeits- und Effekt-Analyse (FMEA) usw. lassen sich beispielsweise die Voraussetzungen für die Zertifizierung eines Qualitätssicherungssystems bzw. für den Aufbau eines Total Quality Management erfüllen (vgl. die Beiträge von Petrick und Kamiske/Malorny in diesem Band). Die spezifische Herausforderung für das HRM besteht darin, die jeweils optimale Maßnahmenkombination zu identifizieren. Als eher erklärungsbedürftig erweist sich der Verdrängungsverbund. Er besagt, daB bei Intensivierung bestimmter Führungsinstrumente (z.B. vertrauensbasierte Zusammenarbeit) andere Instrumente (z.B. Kontrollaktivitäten) zurückgefahren werden sollten, weil sie obsolet sind oder durch ihr hohes Niveau die Verbreitung der Neuerung behindern.

\section{B. Bedarfsverbund im Human-Ressourcen-Management}

\section{Personalbedarfe im Produktionsmanagement}

Die konventionelle Ermittlung des qualitativen und quantitativen Bruttobedarfs an Produktionspersonal vollzieht sich in der Abhängigkeitskette „Produktionsprogramm - Tech- 
nik - Organisation - Personal" ${ }^{\text {" }}$ Im Vordergrund der qualitativen Bedarfsermittlung steht die Fachkompetenz (Berufsbilder für Facharbeiter und Ingenieure). Der Bedarf an Methodenund Sozialkompetenz wird meist nur nachrangig bzw. implizit berücksichtigt. Technikund Organisationsgegebenheiten sind in der quantitativen Bedarfsrechnung zu Kennzahlen (Arbeitskoeffizienten) verdichtet, die gegebenenfalls durch einzusparende Ratio-Vorgaben korrigiert werden. Im Falle der Stellenplanmethode erfolgt die Personalbemessung explizit unter Rückgriff auf organisatorische Gegebenheiten, konkret auf Stellenpläne im Verbund mit Betriebszeiten- und Arbeitszeitenregelungen (Schichtsysteme, Voll- bzw. Teilzeitbeschäftigung usw.)

Organisationsinduzierte Personalbedarfe dominieren bei der Bemessung des Gemeinkostenpersonals im Produktionsbereich. Der Bedarf an Führungskräften (Center-Leiter, Meister, Koordinatoren usw.) läßt sich mit Hilfe von organisatorisch determinierten Leitungsspannen bzw. Gruppengrößen ermitteln. Hier zeichnet sich ein Trend zur Ausdehnung der Leitungsspannen ab. Auch der Bedarf an Unterstützungskräften bei den Sekundärfunktionen „Instandsetzung ${ }^{4},{ }_{n}$ Arbeitsvorbereitung ${ }^{\prime},{ }_{\text {}}$ Qualitätssicherung ${ }^{\prime}$ und

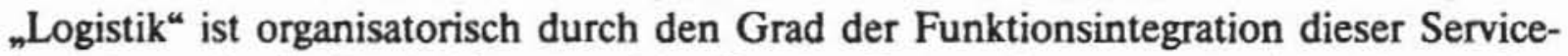
funktionen in die Produktionsbereiche (Inseln, Center, Segmente usw.) bestimmt. Der Bedarf an Reservepersonal (Springer, Vertretungen usw.) wird meist unter Rückgriff auf Fehlzeitenquoten berechnet. Fehlzeiten sind ihrerseits teilweise organisationsbedingt, weil die Arbeitszufriedenheit auch mit organisatorischen Parametern wie z.B. dem Handlungs- und Interaktionsspielraum einer Stelle in Verbindung steht.

\section{Organisationsbedarfe im Produktionsmanagement}

Zusammen mit dem Produktionsprogramm und der Produktionstechnik bestimmen Belegschaftsmerkmale nach dem Prinzip "Organisation folgt Personal ${ }^{\mu}$ den Bedarf an (Re-)Strukturierung der Produktionsorganisation. Gemeinhin beeinflußt die Belegschaftgröße (eines Produktionstandorts oder Cost-Centers) bei unveränderten Leitungsspannen die Anzahl der erforderlichen Hierarchie-Ebenen. Eine ${ }_{n}$ schlanke" Produktionshierarchie

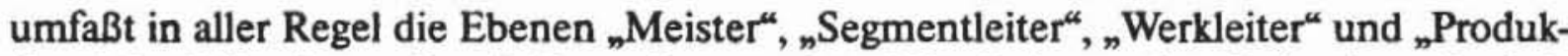
tionsleiter" (im Stammhaus). Desweiteren gehen Mitarbeitermerkmale in eine mitarbeitergerechte Stellenbildung ein, beispielsweise in individualisierte Regelungen für die Übernahme von Verantwortung und Kompetenzen bei Arbeitsanreicherung. Außerdem muß die vorhandene Sozialkompetenz (einschließlich Sprachbarrieren bei hohem Anteil von ausländischen Werkern) bei der Entscheidung „Einzelarbeit oder Gruppenarbeit“ Beachtung finden. Bei High-Tech-Produktion ist die Einrichtung einer Fachlaufbahn für Fertigungsspezialisten überprüfenswert. 


\section{Ergänzungsverbund im Human-Ressourcen-Management}

\section{Entgeltpolitik}

Das Leistungspotential eines Mitarbeiters ergibt sich als Produkt aus Leistungsbereitschaft (Motivation) und Leistungsfähigkeit (Qualifikation). Die Motivation ist ihrerseits ein Produkt aus Motiven (Existenz-, Sozial- und Wachstumsmotive), Valenzen (Anreizwerten) und kognitiven Erfolgserwartungen (z.B. sogenannten Instrumentalitäten). Die $\mathrm{An}$ reizpolitik bildet ein gemeinsames Betätigungsfeld von Personal- und Organisationsarbeit. Die Organisationsgestaltung steuert hier insbesondere intrinsische Anreize durch motivierende Stelleninhalte bei. Das breite Spektrum der extrinsischen Anreize, einschlieBlich der traditionsreichen Entgeltpolitik (vgl. Abbildung 5), liegt eher im Zuständigkeitsbereich des Personalmanagements, wobei allerdings von der jeweiligen Organisationsform ein spürbarer Einfluß auf die Arbeitswerte ausgeht.

Das Direktentgelt eines Mitarbeiters setzt sich stets aus anforderungsgerechtem Grundentgelt, leistungsgerechter Zulage bzw. Prämie und bedürfsnisgerechten Entgeltbestandteilen (Sozialprinzip) zusammen, wobei die Arbeitsmarktverhältnisse (z.B. Facharbeiter mit Programmierungskenntnissen) als Niveaufaktor für die Marktgerechtheit der Entgeltbemessung sorgen. Die aktuelle Auseinandersetzung mit leistungsgerechter Entlohnung (vgl. Abbildung 5) kreist um Fragen der Arbeitsbewertung bei Übernahme von Sekundärfunktionen durch Teams, der Honorierung von kooperativem Verhalten durch Gruppenprämien (kollektive Leistungsbewertung), der Gratifikation von Vorschlägen im Rahmen eines kontinuierlichen Verbesserungsprozesses (Kaizen) und um die Entlohnung von vorgehaltenem Leistungspotential, auch wenn dies nicht effektiv genutzt wird (Qualifikationslohn).

\section{Kooperation}

Auch die Optimierung der Zusammenarbeit im Produktionsbereich erfordert ein simultanes Engagement von Organisationsarbeit und Personalarbeit. Herkömmlich werden Fragen der Kooperation mit der Bestimmung eines adäquaten Führungsstils (z.B. in der Meisterwirtschaft) gleichgesetzt (vgl. Abbildung 6).

Im Zuge einer verstärkten Autonomisierung der Produktion erweist sich die Führungsperspektive als zu eng. Bei segmentierter Fertigung kristallisieren sich insgesamt drei Kooperationsarenen heraus, für die Spielregeln der Zusammenarbeit gefunden werden müssen. Erstens geht es um die Zusammenarbeit innerhalb einer Insel bzw. eines teilautonomen Teams (Arbeitsverteilung, Zeithoheit, Wahl des Teamsprechers usw.), zweitens um die Zusammenarbeit zwischen Teams über die Wertschöpfungskette und drittens um die Einbindung der Center in die Produktionsplanung (Zielvereinbarungen) sowie die Kontakte zu zentralen Service-Bereichen (z.B. Ermittlung von Verrechnungspreisen).

Kooperation und Führung erschöpft sich nicht im Verfassen von Kooperations- und Führungsgrundsätzen. Sie erstreckt sich auch auf die Qualifizierung der Kooperations- 


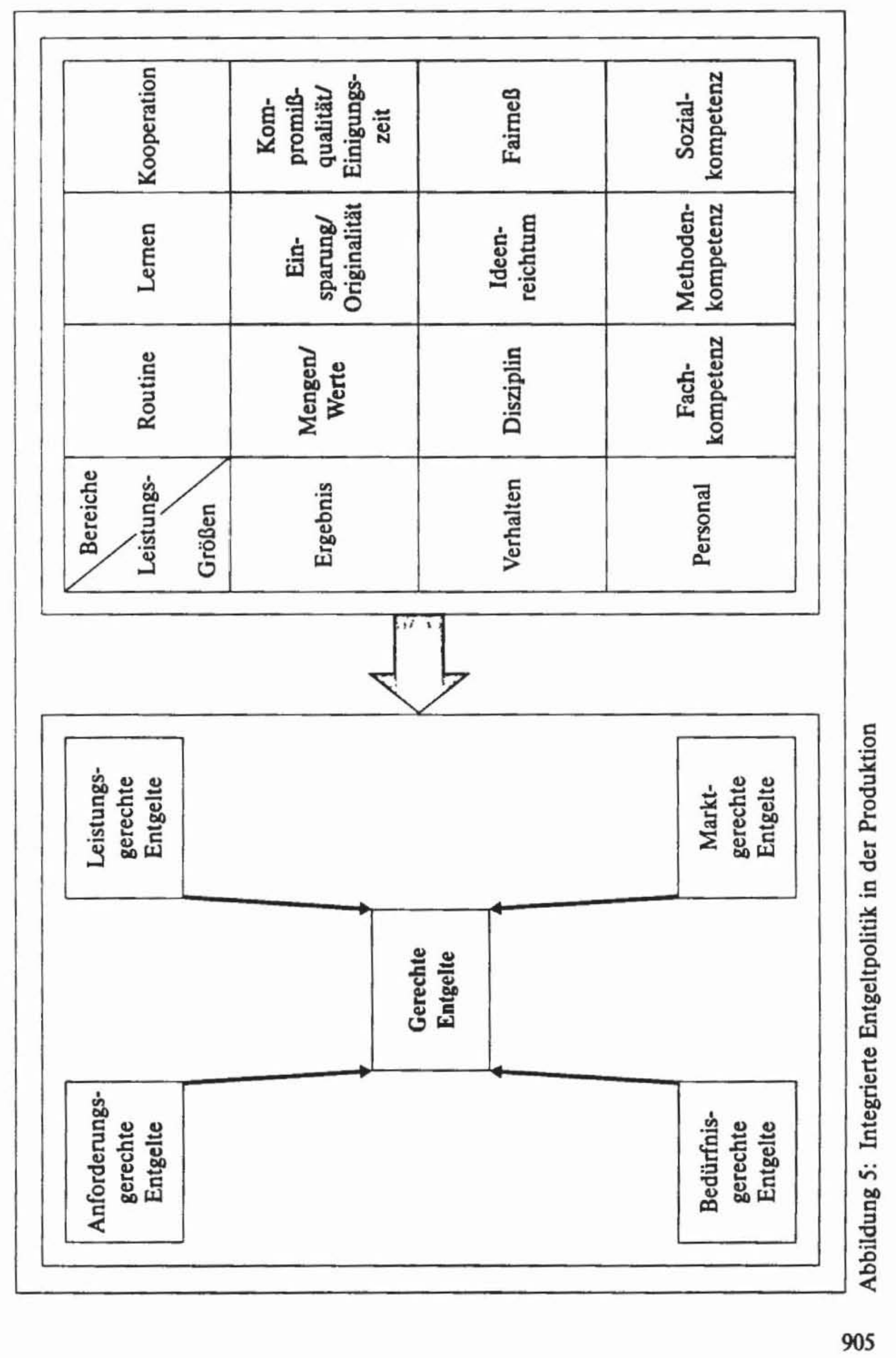




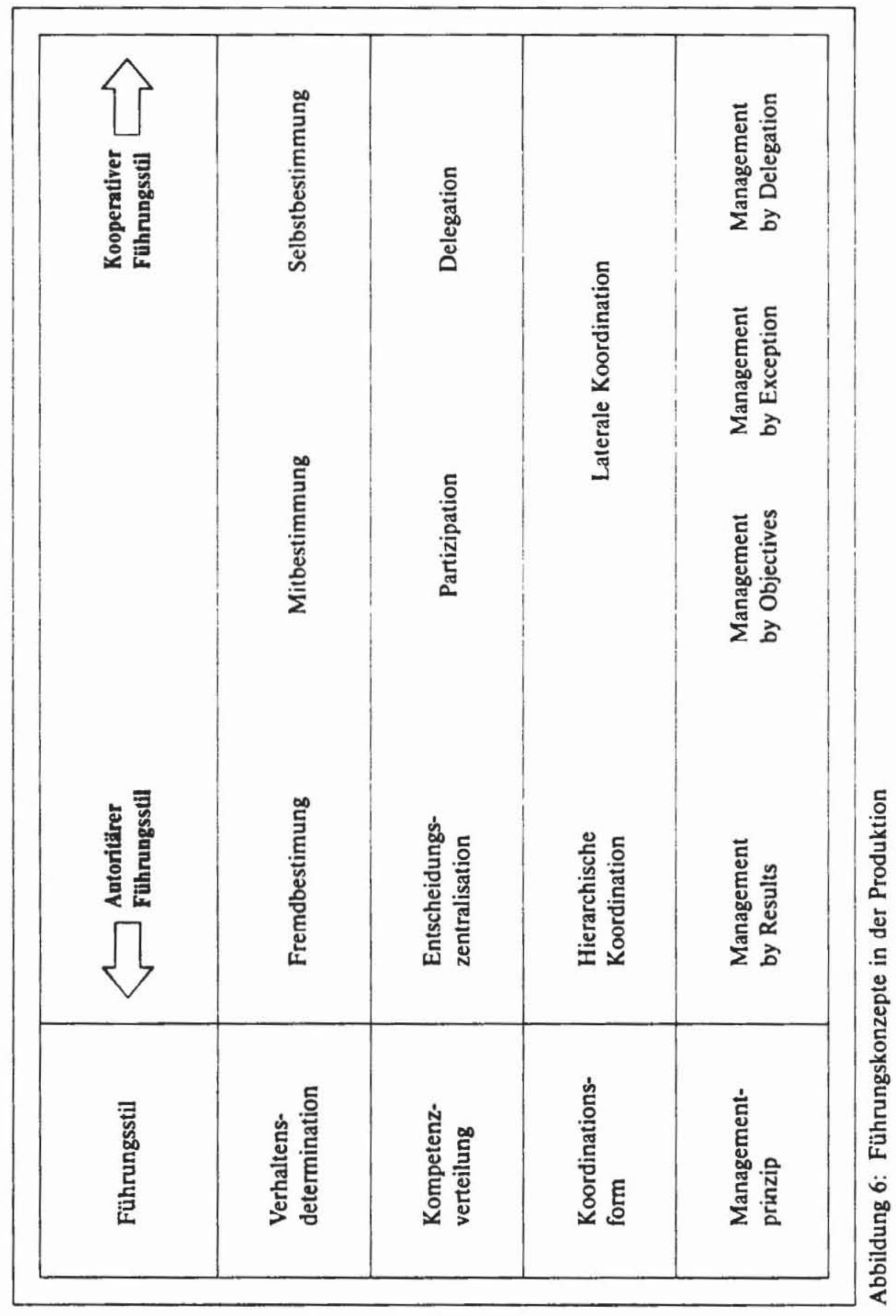


partner und Führungskräfte, etwa für Aufgaben im Konfliktmanagement. Hierzu zählt auch die Vorbereitung des Meisters bzw. Gruppensprechers auf seine Rolle als Moderator bzw. Schlichter.

\section{Unternehmertum}

Wettbewerbsstrategien (Scale-, Scope- und Speed-Vorteile, Differenzierung durch Qualität usw.) müssen nicht nur durch Marketing und F\&E, sondern auch durch die Produktion unterstützt werden (vgl. die Beiträge von Corsten/Will und Zahn in diesem Band). Gemäß dem Prinzip "Structure follows Strategy ${ }^{\prime}$ ist dazu eine ergebnisorientiert unternehmerische Ausrichtung aller Produktionsbereiche erforderlich. Eine unternehmerisch strukturierte Produktion (Stichworte: ${ }_{n}$ Fabriken in der Fabrik ${ }^{4},{ }_{n}$ fraktale Fabrik ${ }^{4},{ }_{n}$ Intrapreneurship ${ }^{4}$ ) ruht auf drei Säulen. Die Organisation muß die verschiedenen Center abgrenzen und nach dem Kongruenzprinzip die passende Center-Variante (Verantwortung für direkte Kosten, Erlöse, Produktivität, Qualität, Termine usw.) bestimmen. Der Personalarbeit obliegt es, geeignete Unternehmerpersönlichkeiten als Center-Leiter zu rekrutieren und zu schulen. Einschlägige Trainingsprogramme konzentrieren sich auf eine kaufmännische Schulung von Technikern. Schließlich muß das Leistungsprogramm für jede unternehmerische Einheit (Tiefe und Breite, Bestimmung der internen und externe Kunden usw.) fixiert werden.

\section{Ideenmanagement}

Das Management von Innovationen und Verbesserungen im Produktionsbereich setzt an Produktionsprozessen und Produktionsressourcen (Fabrikplanung, Verfahrenstechnik, Standortplanung etc.) an. Der Brückenschlag zu neuen Produktideen wird beispielsweise im Rahmen eines Simultaneous Engineering bewerkstelligt. Die Förderung der Ideenfindung erfolgt parallel durch Anreizsysteme (Vorschlagswesen, „Awards“, Wettbewerbe usw.) und durch geeignete Organisationsformen. Verbesserte Produktionsanlagen können zum einen durch Spezialisten aus Stabsabteilungen entwickelt werden, die abgekoppelt von sonstigen Aufgaben unter Laborbedingungen arbeiten. Das Projektmanagement mit zeitweiser Freistellung von Linienmitarbeitern stellt einen zweiten organisatorischen Rahmen für das Ideenmanagement dar. Mit der Zirkelarbeit wird der Verbesserungsprozeß näher an das Alltagsgeschäft verlagert. Eine hochgradige Integration ist erreicht, wenn Ideen sowohl personell als auch zeitlich und räumlich "on the job" entwickelt werden (Kaizen-Prinzip).

\section{Rationalisierung}

${ }_{n}$ Rationalisierung ${ }^{4}$ fungiert als Sammelbezeichnung für alle effizienzsteigernden Maßnahmen des Produktivitäts-, Kosten-, Speed- bzw. Lean Managements. Alle abgestimmten Bemühungen der Organisations- und Personalarbeit um Ratio-Potentiale bauen auf 


\begin{tabular}{|l|l|l|}
\hline $\begin{array}{l}\text { Rationalisierungs- } \\
\text { modus }\end{array}$ & $\begin{array}{l}\text { Rationalisierungs- } \\
\text { basis }\end{array}$ & Beispiele \\
\hline (T)erminieren & $\begin{array}{l}\text { Verbesserung } \\
\text { der Lage }\end{array}$ & $\begin{array}{l}\text { Wahl günstiger Zeitpunkte; Frühzeitig- } \\
\text { keit; Rechtzeitigkeit; proaktive } \\
\text { Intervention }\end{array}$ \\
\hline Ro(u)tinisieren & $\begin{array}{l}\text { Verkürzung } \\
\text { der Länge }\end{array}$ & $\begin{array}{l}\text { Übungs- bzw. Lerneffekte; Vermeiden } \\
\text { von Medienbrüchen; Radikales Unter- } \\
\text { lassen; Stichprobenweises Durchführen; } \\
\text { konditionalisiertes Durchführen; Ver- } \\
\text { einfachen }\end{array}$ \\
\hline Komprimiere(n) & $\begin{array}{l}\text { Verkürzung } \\
\text { der Abstände }\end{array}$ & $\begin{array}{l}\text { Zeitliche Verdichtung; Synchronisieren; } \\
\text { Überlappen; Simultanisieren; Paralle- } \\
\text { lisieren; Prinzip der kurzen Wege }\end{array}$ \\
\hline Pr(i)orisieren & $\begin{array}{l}\text { Verbesserung } \\
\text { der Abfolgen }\end{array}$ & Prioritätsregeln; Sukzession der Planung \\
\hline Rythmisiere(n) & $\begin{array}{l}\text { Verbesserung } \\
\text { der Abstandsmuster }\end{array}$ & $\begin{array}{l}\text { Verstetigen; GleichmäBigkeit; Regel- } \\
\text { mäßigkeit }\end{array}$ \\
\hline (G)ruppieren & $\begin{array}{l}\text { Verbesserung } \\
\text { der Abfolgemuster }\end{array}$ & $\begin{array}{l}\text { Losgrößenbildung; Stapeln; Blocken; } \\
\text { Sortieren; Repetitives Abarbeiten }\end{array}$ \\
\hline
\end{tabular}

Abbildung 7: Ablauforganisatorische Rationalisierung

einer prozeßorientierten Sichtweise der Produktion auf. Die klassischen Ansätze für eine Rationalisierung stammen weitestgehend aus der Ablauforganisation (vgl. Abbildung 7 ).

Durch das Prinzip der Kundenorientierung wurde die technische Sichtweise von Produktionsprozessen im Rahmen eines Geschäftsprozeßmanagements auf das Management der gesamten Auftragsabwicklung ausgedehnt. Zur Prozeßunterstützung kommt neben einer segmentierten Produktion (Prinzip der kurzen Wege) vor allem die Einrichtung von zusätzlichen Stellen mit Auftragsverantwortung (Auftragszentren, Vertriebsinseln, Termin- bzw. ProzeBverantwortliche etc.) in Betracht.

Neben der Beherrschung von Routineprozessen verlangt effiziente Produktion auch eine optimierte Kopplung von routinisierten Abwicklungsprozessen (Lieferzyklen) und Entwicklungsprozessen (Innovationszyklen). Diese erfolgskritische Schnittstelle läßt sich in einem Simultaneous Engineering handhaben, in welches unter anderem die Anforderungen des Produktionsbereichs bezüglich fertigungs- bzw. montagegerechter Konstruktion eingehen. Flankierend müssen an dieser Schnittstelle Bemühungen um eine Anlaufbeschleunigung sowie ein Speed-Änderungsmanagement ansetzen, besonders bei Einzelfertigung bzw. kleinen Losgrößen. 


\section{Technologiefolgen-Management}

Die Potentiale von intelligenten Produktions- und Logistiktechnologien aus der Mikroelektronik lassen sich nur voll ausschöpfen, wenn Technik, Organisation und Personal wechselseitig aufeinander abgestimmt werden. Für ein HRM resultiert hieraus die Aufgabe, die Technologiefolgen von FFS, Roboterisierung, hierarchischem Rechnerverbund usw. auf die (1) Arbeitssysteme in der Produktion, auf die (2) Produktionsorganisation und auf das (3) Produktionspersonal zu ermitteln und mit dem Anliegen einer optimalen Nutzung der Human-Ressourcen in Einklang zu bringen (vgl. Abbildung 8). Diesem Zweck dienen vornehmlich technologieinduzierte Qualifizierungsoffensiven, Anpassungen der Berufsbilder und Bemühungen um eine menschengerechte Technikgestaltung.

Bei der Abschätzung und Handhabung von Technologienfolgen müssen HR-Manager mehrdeutige Abhängigkeiten einkalkulieren. Die neuen Technologien determinieren keinesfalls einen bestimmten Zentralisationsgrad der Arbeitsorganisation, sondern sind mit unterschiedlichen Graden der (De-)Zentralisation vereinbar. Unklar ist auch, in welchen Fällen die Einführung intelligenter Technologien zu einer Höherqualifizierung, Dequalifizierung oder Polarisierung in den Qualifikationsstrukturen führt. Kontrovers bleibt auch die Frage des technikgerechten Entgeltsystems im "magischen Dreieck“" von Zeitlohn, Prämienlohn und Qualifikationslohn.

\section{Verdrängungsverbund im Human-Ressourcen-Management}

Das optimale Management von Human-Ressourcen läuft nicht in allen Fällen auf eine komplementäre Intensivierung von personalpolitischen und organisatorischen Gestaltungsmaßnahmen hinaus. Gerade im Zusammenhang mit der eindrucksvollen Performance von japanischen Produktionssystemen wird behauptet, daß man das Investment in sogenannte weiche Führungsfaktoren (Qualifikation, Kultur usw.) intensivieren, dafür aber die sogenannten harten Führungsfaktoren in Gestalt von organisatorischen Reglementierungen (zentrale PPS, Qualitätssicherung usw.) reduzieren solle. Analog sei es beispielsweise wichtiger, daß ein Meister durch persönliche Autorität (soziale Kompetenz, fachliche Expertise, Informationsvorsprung usw.) führe und weniger auf der Basis seiner Positionsmacht.

Eine äußerst relevante Erscheinungsform des Verdrängungsverbunds bildet die Ersetzung von Fremdorganisation durch Selbstorganisation. Ein erster Schritt in diese Richtung bildet der Übergang vom Prinzip der (Ziel-)Vorgabe auf das Prinzip der (Ziel-)Vereinbarung (für Arbeitspensen, Service-Leistungen, Verrechnungspreise). Ein weiterer produktionsspezifischer Schritt ist die Ersetzung von Fremdprüfung durch Selbstprüfung im Qualitätswesen sowie die Übertragung von "Souveränität“ in Sachen Zeitplanung, Bestimmung des Teamsprechers usw. auf die teilautonomen Gruppen. 


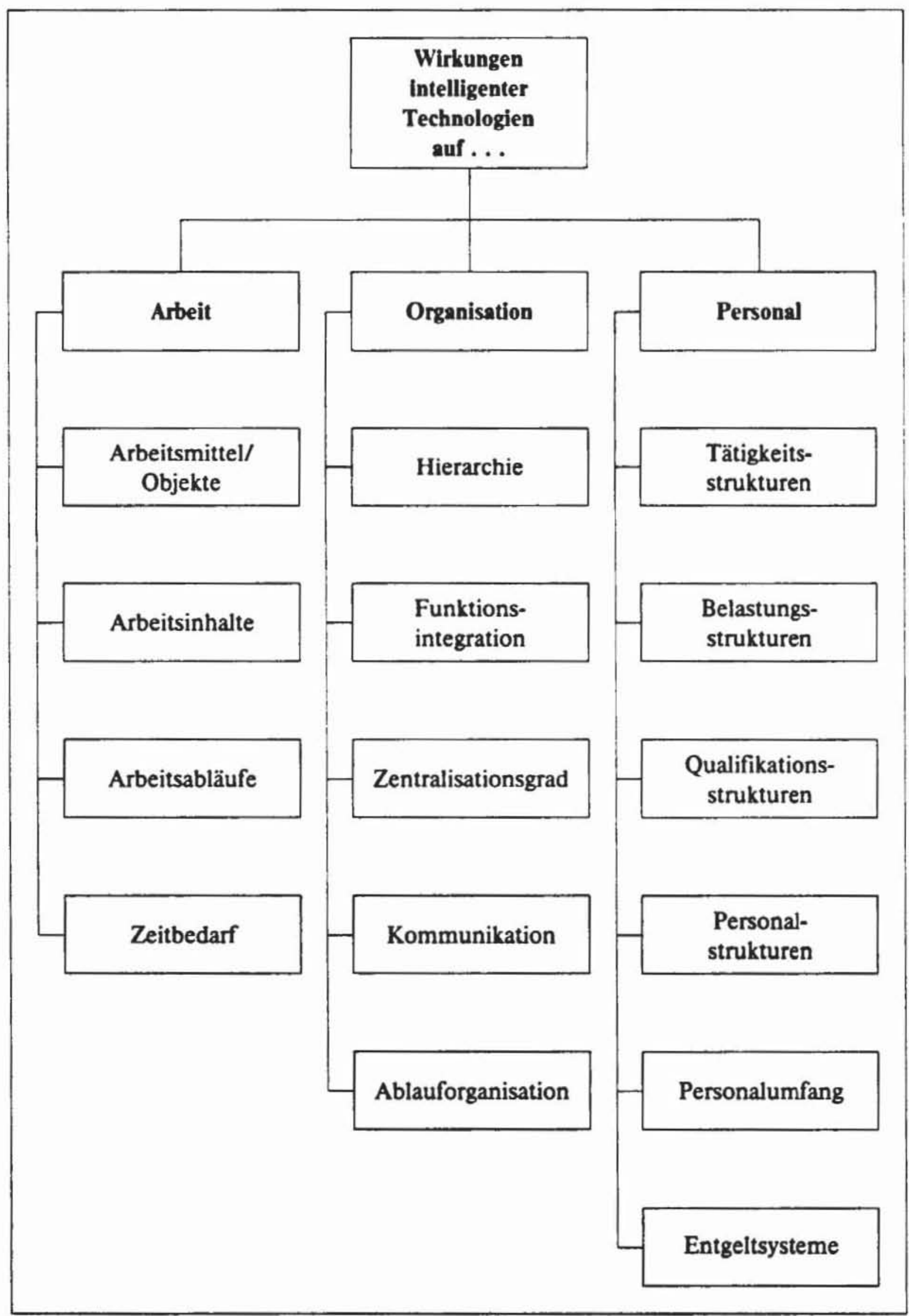

Abbildung 8: Technologiefolgen-Management durch HRM 
Selbstorganisation setzt voraus, daß Organisationskompetenz vor Ort verfügbar ist. Die Förderung der Fähigkeiten zur Selbstanpassung und Selbstabstimmung muß von der Personalarbeit aktiv unterstützt werden. Diesem Anliegen dienen Kurse in Selbstmanagement, die Rekrutierung von anpassungs- und kooperationsfähigen Mitarbeitern sowie Entlohnungssysteme, die Gemeinschaftleistungen (von Gruppen und Abteilungen) stärker honorieren.

\section{Literatur}

Bokranz, R.: Einführung in die Arbeıtswissenschaft. Analyse und Gestaltung von Arbeitssystemen, Stuttgart 1991. BUhNer, R.: Personalentwicklung für neue Technologien in der Produktion, Stuttgart 1986.

BUhNER, R.: Betriebswirtschaftliche Organisationslehre, 6. Aufl., München/Wien 1992.

CoNRAD, P.: Human Resource Management - eine „lohnende“ Entwicklungsperspektive?, in: Zeitschrift für Personalforschung, 5. Jg. (1991), S. 411-445.

Corsten, H.: Produktionswirtschaft, 3. Aufl., München/Wien 1992.

Eidenmuller, B.: Die Produktion als Wettbewerbsfaktor, Zürich/Köln 1989.

Famularo. J. (Hrsg.): Handbook of Human Resource Administration, 2. Aufl., New York u.a. 1985.

FrESE, E.: Industrielle Personalwirtschaf, in: Industriebetriebslehre, hrsg. v. M. Schweitzer, München 1990, S. 219329.

Frese, E.: Grundlagen der Organisation, 5. Aufl., Wiesbaden 1991.

Hentze, J.: Personalwirtschaftslehre, Bd. 1 u. 2, 5. Aufl., Bern u.a. 1991.

Kreikebaum, H.: Industrielle Unternehmungsorganisation, in: Industriebetriebslehre, hrsg. v. M. Schweitzer, München 1990, S. 147-218.

MAG, W.: Einführung in die betriebliche Personalplanung, Darmstadt 1986.

MArR, R.; Strtzel, M.: Personalwirtschaft: Ein konfliktorientierter Ansatz, München 1979.

Oechsler, W.: Personal und Arbeit. Einführung in die Personalwirtschaft, 4. Aufl., München u.a. 1992.

Pfeiffer, W.; Dörrae, U.; Stoll, E.: Menschliche Arbeit in der industriellen Produktion, Göttingen 1977.

REIss, M.: Personalarbeit statt Organisationsarbeit? Selbstorganisation als Herausforderung für Personalmanagement und Organisationsgestaltung, in: Moderne Industriegesellschaft, hrsg. v. M. v. Hauff, Ludwigsburg/Berlin 1991, S. 223-241.

RKW (Rationalisierungs-Kuratorium der Deutschen Wirtschaft e.V.): Handbuch Praxis der Personalplanung. 2. Aufl., Neuwied u.a. 1988.

Schanz, G. (Hrsg.): Handbuch Anreizsysteme, Stuttgart 1991.

Scholz, C.: Personalmanagement, 2. Aufl., München 1991.

StARHLE, W.: Human Resource Management (HRM), in: ZfB, 58. Jg. (1988), S. 576-587.

Staehle, W.: Management, 6. Aufl., München 1991.

WAGNER, D.: Organisation, Führung und Personalmanagement, Freiburg 1989. 